\title{
ChemComm
}

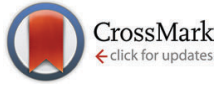

Cite this: Chem. Commun., 2015, 51, 9137

Received 15th March 2015, Accepted 27th April 2015

DOI: $10.1039 / c 5 c c 02197 a$

www.rsc.org/chemcomm

\section{Imaging the lipid bilayer of giant unilamellar vesicles using red-to-blue light upconversion $\dagger$}

\author{
Sven H. C. Askes, $\ddagger^{a}$ Néstor López Mora, $\ddagger^{a}$ Rolf Harkes, ${ }^{b}$ Roman I. Koning, ${ }^{c}$ \\ Bram Koster, ${ }^{c}$ Thomas Schmidt, ${ }^{b}$ Alexander Kros ${ }^{a}$ and Sylvestre Bonnet*a
}

\begin{abstract}
Red-to-blue triplet-triplet annihilation upconversion was obtained in giant unilamellar vesicles. The upconverted light was homogeneously distributed across the membrane and could be utilized for the imaging of individual giant vesicles in three dimensions. These results show the great potential of TTA-UC for imaging applications under anoxic conditions.
\end{abstract}

Upconversion luminescence (bio)imaging offers great advantages over conventional imaging. The absence of auto-fluorescence results in high contrast images, while photons of low energy, i.e. within the phototherapeutic window (600-1000 nm), afford higher tissue penetration and negligible irradiation damage. For these reasons lanthanoid-based upconverting nanoparticles (UCNPs), for example, have attracted much interest. ${ }^{1,2}$ However, UCNPs suffer from several disadvantages, such as the need for high excitation power, the low absorption cross section of lanthanoid ions, and low upconversion efficiency in aqueous solution (typically $\leq 0.5 \%)^{2}{ }^{2}$ In contrast, triplet-triplet annihilation upconversion (TTA-UC) requires low excitation power $\left(<100 \mathrm{~mW} \mathrm{~cm}^{-2}\right)$, employs sensitizers having high extinction coefficients in the phototherapeutic window, and has achieved upconversion quantum yields up to $14 \%$ in aqueous solution., ${ }^{2,3}$

In TTA-UC, low-energy photons are converted into higherenergy photons by means of a photophysical mechanism involving a couple of molecular dyes called the sensitizer and annihilator (see Fig. S1, ESI † for a qualitative Jablonski diagram) ${ }^{4-8}$ The sensitizer absorbs the low-energy light, undergoes intersystem crossing (ISC) to a triplet state, and transfers its energy to the annihilator molecule by triplet-triplet energy transfer. Further collision of two triplet

\footnotetext{
${ }^{a}$ Leiden Institute of Chemistry, Leiden University, Einsteinweg 55, 2333 CC Leiden, The Netherlands. E-mail: bonnet@chem.leidenuniv.nl

${ }^{b}$ Leiden Institute of Physics, Leiden University, Niels Bohrweg 2, 2333 CA Leiden, The Netherlands

${ }^{c}$ Leiden University Medical Center, Einthovenweg 20, 2333 ZC Leiden, The Netherlands

$\dagger$ Electronic supplementary information (ESI) available: Supplementary information, and Video V1. See DOI: 10.1039/c5cc02197a

\$ These authors contributed equally to this work.
}

annihilator molecules leads to triplet-triplet annihilation (TTA), whereby one annihilator molecule is promoted to the excited singlet state, whereas the other one falls back to the ground state. The singlet annihilator returns to the ground state by emission of a high-energy photon, thus realizing upconversion. Most molecular dyes used in TTA-UC are highly lipophilic and require supramolecular strategies to be used in aqueous solution. ${ }^{9-14}$ For example, sub-micrometer sized TTA-UC particles have been proposed for in vitro or in vivo imaging., ${ }^{2,11,12}$ We now demonstrate that TTA-UC can also be used for the imaging of lipid membranes.

Giant Unilamellar Vesicles (GUVs) are classical tools in fluorescence imaging, as their large size (1-100 $\mu \mathrm{m}$ diameter) allows for direct observation of individual vesicles by optical microscopy techniques. ${ }^{15}$ GUVs have for example been used for visualizing lipid rafts, membrane fusion, or ion transport. ${ }^{16}$ In this study we functionalized PEGylated GUVs with palladium tetraphenyltetrabenzoporphyrin (1) as photosensitizer and perylene (2) as the annihilator (Fig. 1a), and studied red-to-blue TTA-UC in the membrane of the vesicles by optical microscopy. The aim of the study was to investigate the dye distributions across the membrane, the homogeneity of upconverted emission in the lipid bilayer, and the upconversion stability under imaging conditions. The growth of high-quality giant vesicles with a well-defined shape in physiologically relevant conditions, i.e., at high ionic strengths, was until recently considered as a challenge, but a new method was recently developed by some of us that is compatible with such conditions (up to $\left.320 \mathrm{mOsm} \mathrm{kg}^{-1}\right){ }^{17}$

Upconverting giant vesicles GUV12 were thus prepared from a lipid mixture of $95 \mathrm{~mol} \%$ phospholipid (either 1,2-dimyristoylsn-glycero-3-phosphocholine, i.e. DMPC, or 1,2-dioleoyl-sn-glycero3-phosphocholine, i.e. DOPC), $4 \mathrm{~mol} \%$ sodium $\mathrm{N}$-(carbonylmethoxypolyethylene glycol-2000)-1,2-distearoyl-sn-glycero-3-phosphoethanolamine (DSPE-MPEG-2000), $0.5 \mathrm{~mol} \%$ compound 2, and $0.02 \mathrm{~mol} \%$ compound 1 . The complete procedure is described in the ESI. $\dagger$ Briefly, the dye-containing lipid mixture in chloroform was deposited on a chemically cross-linked dextran-poly(ethylene glycol) hydrogel substrate, dried to form 


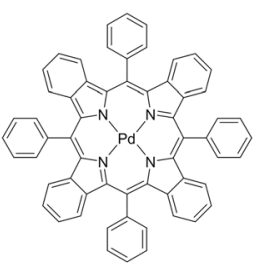

1

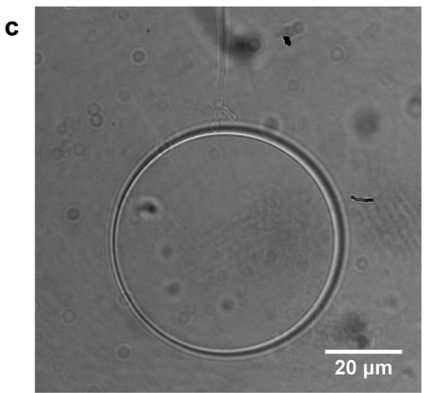

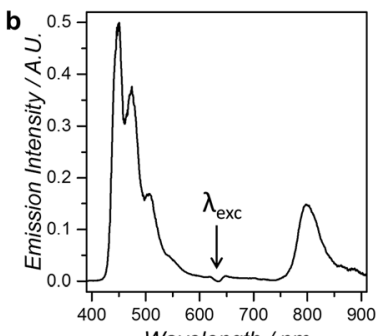

Wavelength $/ \mathrm{nm}$

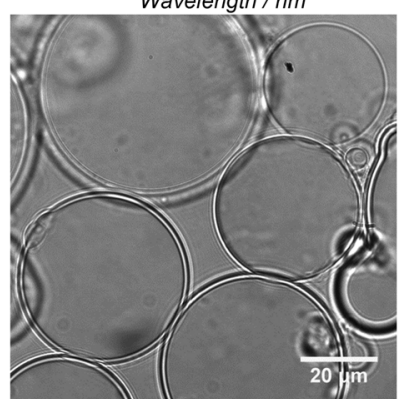

Fig. 1 (a) Chemical structures of palladium tetraphenyltetrabenzoporphyrin (1) and perylene (2). (b) Emission spectra of DOPC upconverting GUVs with $30 \mathrm{~mW} 630 \mathrm{~nm}$ excitation $\left(0.24 \mathrm{~W} \mathrm{~cm}^{-2}\right.$ intensity) at $298 \mathrm{~K}$ in sulfitesupplemented (0.3 M) PBS buffer under air. (c) Bright field micrographs of DOPC (left) and DMPC (right) upconverting giant vesicles at $298 \mathrm{~K}$.

a lipid film, and then the film was re-hydrated with phosphate buffered saline (PBS) supplemented with $0.3 \mathrm{M}$ sodium sulfite $\left(\mathrm{Na}_{2} \mathrm{SO}_{3}\right)$ and $0.2 \mathrm{M}$ sucrose at 293-308 $\mathrm{K}$. Transferring the solution onto a microscopy slide allowed for bright field imaging on a custom-build microscope based on an inverted microscopy setup. The images (Fig. 1c) confirmed that for both lipid compositions (DMPC or DOPC) free-floating single vesicles were obtained, together with clusters of smaller vesicles. The images also show that the self-assembled vesicles were giant (diameter 1-100 $\mu \mathrm{m}$ ), unilamellar, and spherical. The fact that almost identical procedures can be employed for preparing GUVs from lipids having a marked difference in their gel-toliquid transition temperature $\left(T_{\mathrm{m}}=-17.3{ }^{\circ} \mathrm{C}\right.$ and $23.9{ }^{\circ} \mathrm{C}$ for pure DOPC and DMPC, respectively), ${ }^{18}$ demonstrates the flexibility of the GUV preparation method. For comparison, much smaller LUVs (samples LUV12) with an average diameter of $c a$. $150 \mathrm{~nm}$ were prepared from the same lipid mixture but using a standard hydration-extrusion protocol (ESI, $\dagger$ Fig. S2).

Sodium sulfite was added in the buffer as an oxygen-scavenging agent. Since the triplet states involved in TTA-UC are readily quenched by molecular oxygen, it is common practice to deoxygenate samples before measuring upconverted emission. With LUVs de-oxygenation can be achieved by, for example, bubbling the solution with argon or $\mathrm{N}_{2}$. In the case of GUVs imaging however, bubbling an inert gas through the solution would at least impair visualization of single GUVs during a long time period of time due to convection, or even lead to damaging of the giant vesicles, so that supplementing the buffer with an oxygen scavenger is highly preferred. In a preliminary experiment, upconversion emission spectra of LUV12 samples deoxygenated by either argon bubbling for 30 minutes or by adding $0.3 \mathrm{M}$ sodium sulfite to the buffer, were compared (see Fig. S4 and ESI $\dagger$ for details). When irradiated at $630 \mathrm{~nm}$ the emission spectrum of such LUVs at $298 \mathrm{~K}$ shows at $800 \mathrm{~nm}$ the phosphorescence band of 1 , and between 450 and $600 \mathrm{~nm}$ the blue singlet emission from 2 (Fig. S4, ESI $\dagger$ ). The spectra from both deoxygenation methods were found to be very similar. It was thus concluded that $\mathrm{Na}_{2} \mathrm{SO}_{3}$ does not interfere with the photophysical processes at the origin of upconversion, and that sulfite might be used for scavenging dioxygen in a GUVcontaining sample as well.

Indeed, even though addition of $\mathrm{Na}_{2} \mathrm{SO}_{3}$ significantly increased the ionic strength of the buffer (from $278 \pm 1 \mathrm{mOsm} \mathrm{kg}^{-1}$ for PBS buffer to $884 \pm 11$ mOsm kg ${ }^{-1}$ when supplemented with $0.3 \mathrm{M}$ sodium sulfite), as explained above sodium sulfite did not prevent the assembly of DMPC or DOPC GUV12 using the hydrogel method. No differences in vesicle yield and morphology were observed in presence or absence of sodium sulfite in the buffer. This result demonstrates that the dextran-poly(ethylene glycol) hydrogel substrate is able to produce GUVs at high ionic strength, which is a significant advantage over alternative GUV preparation methods such as electroformation or gentle hydration, which often fail in such conditions. When irradiated at $630 \mathrm{~nm}$ under air, the emission spectrum of the DMPC or DOPC GUV12 samples prepared in a sulfite-supplemented buffer was identical to the emission spectrum of the corresponding LUV12 samples (Fig. 1b and Fig. S4, ESI $\dagger$ ), showing that the dyes 1 and 2 were indeed incorporated in the lipid bilayer.

GUV12 samples were then visualized by emission microscopy at $298 \mathrm{~K}$ (Fig. 2 and ESI $\dagger$ ). When the vesicles were illuminated with violet light $(405 \mathrm{~nm})$, i.e. by direct excitation of perylene (2), fluorescence was clearly detected at the membrane (Fig. 2b). To visualize upconversion, a $630 \mathrm{~nm}$ continuous wave PDT laser was coupled into the microscope and set at a power of a few milliwatts, resulting in the focal spot in an intensity of $\sim 300 \mathrm{~W} \mathrm{~cm}^{-2}$. All wavelengths other than $450-575 \mathrm{~nm}$ were strictly blocked by a combination of notch and short-pass filters (ESI $\dagger$ ). High-quality images were obtained that were superimposable to the bright field images and to the fluorescence images recorded under white and violet light irradiation, respectively (Fig. 2a-c). Control samples were prepared in which the porphyrin sensitizer 1 was omitted from the formulation (GUV2). Images recorded in identical conditions were black, i.e., no blue emission was observed (Fig. S10, ESI $\dagger$ ). GUV12 samples prepared in absence of sulfite oxygen scavenger and observed under air did not give any observable emission either (Fig. S11, ESI $\dagger$ ). Altogether, these observations prove that the blue images recorded under $630 \mathrm{~nm}$ irradiation of GUV12 samples supplemented with sulfite comes from the TTA upconversion process and are not the result of sensitizer emission (at $800 \mathrm{~nm}$ ) or of two-photon absorption. Overall, all data conclude that both dyes 1 and 2 co-localize in the membrane and result in TTA upconversion. At this scale of observation the upconverted emission is homogeneous across the membrane and no phase separation of the lipids or dyes was observed.

Under the red-light irradiation conditions initially used in the microscopy setup ( $630 \mathrm{~nm}$ at an intensity of $320 \mathrm{~W} \mathrm{~cm}^{-2}$ ), substantial bleaching of the upconverted emission of GUV12 


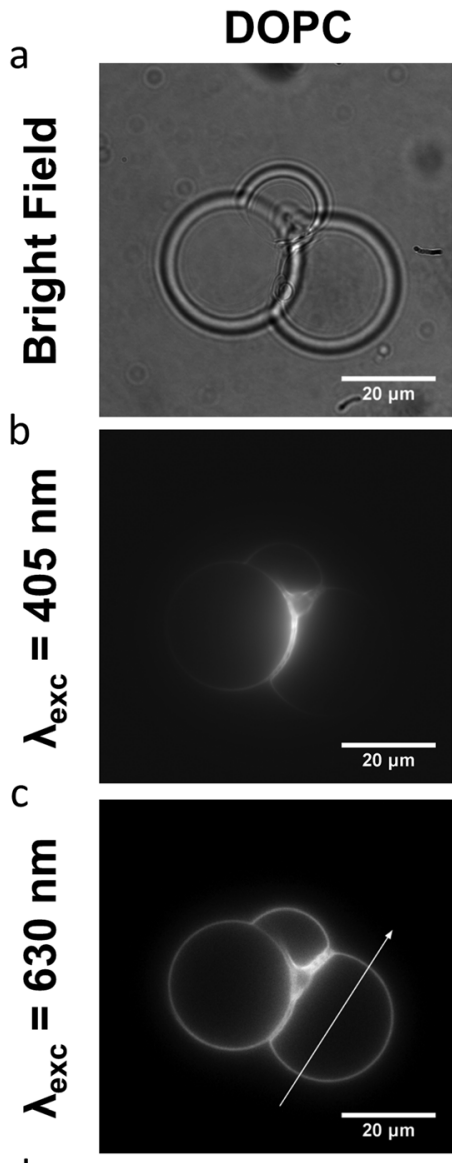

d

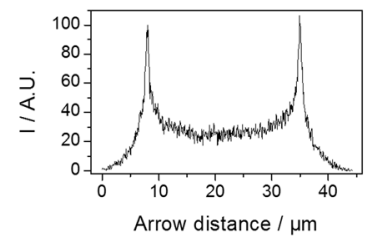

DMPC
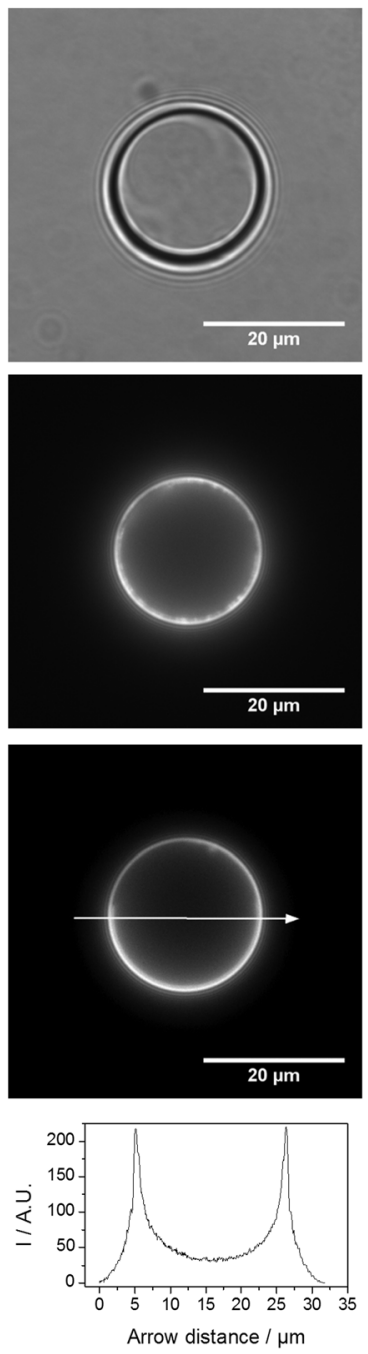

Fig. 2 Imaging of DOPC (left) and DMPC (right) upconverting giant vesicles (GUV12) with (a) bright field, (b) $405 \mathrm{~nm}$ excitation and 450$500 \mathrm{~nm}$ detection, and (c) $630 \mathrm{~nm}$ excitation and 450-575 nm detection. (d) Upconversion intensity profile plot following the arrows in the images directly above (c). At $630 \mathrm{~nm}$ : laser spot size diameter $39 \mu \mathrm{m}$, power $3.8 \mathrm{~mW}$, intensity $320 \mathrm{~W} \mathrm{~cm}^{-2}$. At $405 \mathrm{~nm}$ : laser spot size diameter $22 \mu \mathrm{m}$ (power $1 \mathrm{~mW}$, intensity $60 \mathrm{~W} \mathrm{~cm}^{-2}$ ) for DOPC image or $39 \mu \mathrm{m}$ (power $1 \mathrm{~mW}$, intensity $300 \mathrm{~W} \mathrm{~cm}^{-2}$ ) for DMPC image. Images were acquired at $298 \mathrm{~K}$ in sulfite-supplemented (0.3 M) PBS buffer.

samples was observed even in presence of $0.3 \mathrm{M}$ of sulfite. A plot of the averaged normalized pixel values as a function of red irradiation time shows that the upconverted emission is halved after less than 3 seconds (Fig. 3). When the light intensity was lowered 60 times (i.e., down to $5.2 \mathrm{~W} \mathrm{~cm}^{-2}$ ) clear upconversion images could still be recorded. In such conditions the bleaching rate was significantly lower (Fig. 3), and the time necessary for halving the upconverted emission intensity of a pixel increased to approximately 15 seconds. The upconversion luminescence of LUV12 in a spectroscopy setup could be observed for less than $8 \mathrm{~mW} \mathrm{~cm} \mathrm{~cm}^{-2}$, with linear power dependency above $60 \mathrm{~mW} \mathrm{~cm}{ }^{-2}$ (Fig. S6, ESI $\dagger$ ). Overall, these findings show that

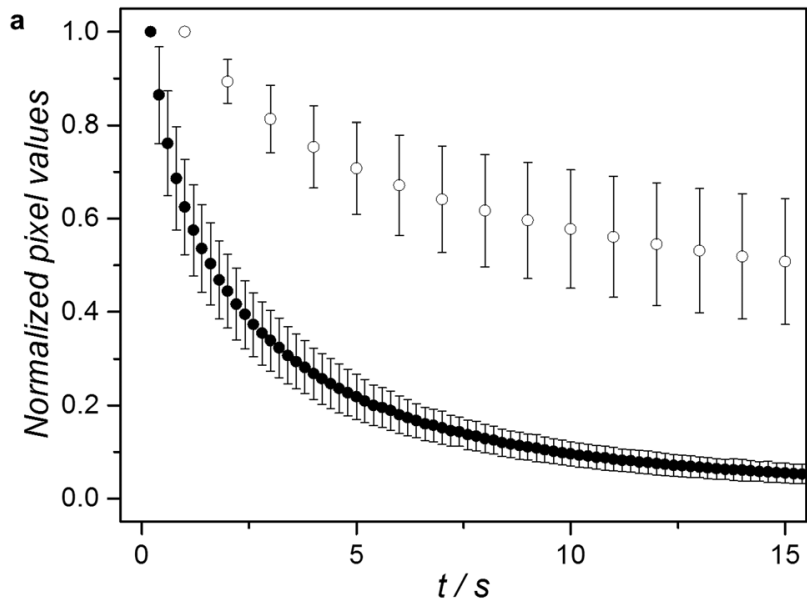

b
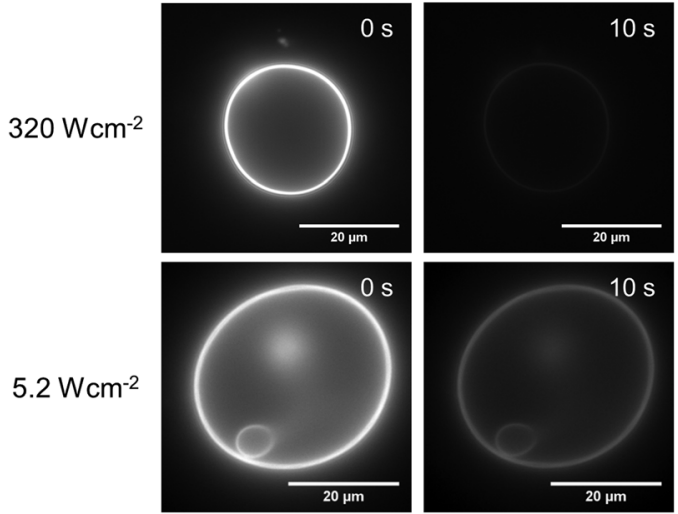

Fig. 3 (a) Averaged normalized pixel values as a function of red irradiation time during upconversion imaging of GUV12 samples in sulfitesupplemented PBS buffer $(0.3 \mathrm{M})$. Conditions: $630 \mathrm{~nm}$ excitation at $320 \mathrm{~W} \mathrm{~cm}^{-2}$ (black filled circles) or $5.2 \mathrm{~W} \mathrm{~cm}^{-2}$ (empty circles), detection in the 450-575 $\mathrm{nm}$ region, $T=298 \mathrm{~K}$. Snapshots were taken with an exposure time of $0.2 \mathrm{~s}\left(320 \mathrm{~W} \mathrm{~cm}^{-2}\right)$ or $1.0 \mathrm{~s}\left(5.2 \mathrm{~W} \mathrm{~cm}^{-2}\right)$. Error bars represent standard deviation based on six individual measurements. (b) Upconversion emission microscopy images of GUV12 samples at $t=0 \mathrm{~s}$ (left) and at $t=10 \mathrm{~s}$ (right) at an illumination intensity of $320 \mathrm{~W} \mathrm{~cm}^{-2}$ (top) and $5.2 \mathrm{~W} \mathrm{~cm}^{-2}$ (bottom). Excitation at $630 \mathrm{~nm}$, detection at $450-575 \mathrm{~nm}$.

high power is not a requirement for the upconversion imaging of GUV12.

In optimized conditions, we realized that the upconverted emission was intense enough to be utilized for reconstructing in $3 \mathrm{D}$ the membrane of the giant vesicles. Z-stack upconversion image acquisition was indeed performed on both DMPC and DOPC GUV12 samples. The illumination intensity was deliberately chosen to be high $\left(320 \mathrm{~W} \mathrm{~cm}^{-2}\right)$ to make sure that z-stack image acquisition was short (200 ms exposure time per slice, $c a$. 45 slices per stack, total acquisition time $<10 \mathrm{~s}$ ). In such conditions, the slight lateral motion of the GUVs did not significantly affect the imaging process. From these stacks, 3D reconstructions were made (e.g. Fig. 4), of which a video was compiled (Video V1 in the ESI $\dagger$ ). This reconstruction demonstrates that the TTA-upconverted emission can be utilized for the three-dimensional reconstruction of an object that is 10 to $30 \mu \mathrm{m}$ in size.

In conclusion, DOPC and DMPC giant vesicles capable of upconverting red light to blue light by means of triplet-triplet 


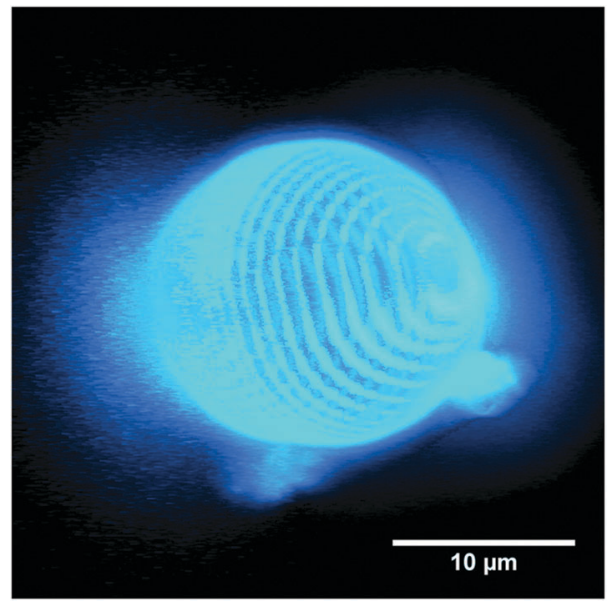

Fig. 4 3D reconstructed image of a DMPC GUV12 sample, rotated counter-clockwise by $50^{\circ}$ about the $y$-axis. Each $z$-slice was imaged at $298 \mathrm{~K}$ with $630 \mathrm{~nm}$ excitation $\left(320 \mathrm{~W} \mathrm{~cm}^{-2}\right.$ ) and detection in the 450$575 \mathrm{~nm}$ region. The $z$-distance between slices was $1.0 \mu \mathrm{m}$. Video V1 (ESI $\dagger$ ) exhibits a $360^{\circ}$ rotational view of this image and of four other individual DMPC and DOPC GUV12.

annihilation were prepared by lipid film hydration on a hydrogel substrate at high ionic strengths. The preparation method is facile and does not involve any specific equipment. Sodium sulfite added as an oxygen scavenger to the vesicle samples allows for observing upconversion even under air. According to optical microscopy, the upconverted emission allows for recording high quality images showing that upconversion is homogeneously realized across the lipid bilayer. The quality and stability of the upconverted images enabled the 3D reconstruction of upconverting GUVs. These results show the great potential of TTA upconversion for imaging applications under anoxic conditions, and open a route towards cell membrane imaging with upconverted light.
Prof. Dr Lies Bouwman is kindly acknowledged for support and scientific discussion. NWO (the Netherlands Organisation for Scientific Research) is acknowledged for a VIDI grant to S.B. The European Research Council is acknowledged for an ERC starting grant to S.B. CONACYT is acknowledged for financial support to N.L.M.

\section{Notes and references}

1 J. Zhou, Q. Liu, W. Feng, Y. Sun and F. Li, Chem. Rev., 2015, 115, 395-465.

2 Q. Liu, W. Feng, T. Yang, T. Yi and F. Li, Nat. Protoc., 2013, 8, 2033-2044.

3 J.-H. Kim and J.-H. Kim, J. Am. Chem. Soc., 2012, 134, 17478-17481.

4 T. N. Singh-Rachford and F. N. Castellano, Coord. Chem. Rev., 2010, 254, 2560-2573.

5 J. Zhao, S. Ji and H. Guo, RSC Adv., 2011, 1, 937-950.

6 Y. C. Simon and C. Weder, J. Mater. Chem., 2012, 22, 20817-20830.

7 Y. Y. Cheng, B. Fückel, T. Khoury, R. 1. G. C. R. Clady, N. J. EkinsDaukes, M. J. Crossley and T. W. Schmidt, J. Phys. Chem. A, 2011, 115, 1047-1053.

8 P. Duan, N. Yanai, H. Nagatomi and N. Kimizuka, J. Am. Chem. Soc., 2015, 137, 1887-1894.

9 A. Turshatov, D. Busko, S. Baluschev, T. Miteva and K. Landfester, New J. Phys., 2011, 13, 083035.

10 M. Penconi, P. L. Gentili, G. Massaro, F. Elisei and F. Ortica, Photochem. Photobiol. Sci., 2014, 13, 48-61.

11 Q. Liu, B. Yin, T. Yang, Y. Yang, Z. Shen, P. Yao and F. Li, J. Am. Chem. Soc., 2013, 135, 5029-5037.

12 C. Wohnhaas, V. Mailänder, M. Dröge, M. A. Filatov, D. Busko, Y. Avlasevich, S. Baluschev, T. Miteva, K. Landfester and A. Turshatov, Macromol. Biosci., 2013, 13, 1422-1430.

13 K. Tanaka, H. Okada, W. Ohashi, J.-H. Jeon, K. Inafuku and Y. Chujo, Bioorg. Med. Chem., 2013, 21, 2678-2681.

14 S. H. C. Askes, A. Bahreman and S. Bonnet, Angew. Chem., Int. Ed., 2014, 53, 1029-1033.

15 P. Walde, K. Cosentino, H. Engel and P. Stano, ChemBioChem, 2010, 11, 848-865.

16 H. Valkenier, N. López Mora, A. Kros and A. P. Davis, Angew. Chem., Int. Ed., 2015, 54, 2137-2141.

17 N. Lopez Mora, J. S. Hansen, Y. Gao, A. A. Ronald, R. Kieltyka, N. Malmstadt and A. Kros, Chem. Commun., 2014, 50, 1953-1955.

18 D. Marsh, Handbook of Lipid Bilayers, Taylor \& Francis Group, LLC, Boca Raton, FL, USA, 2013. 